\title{
PRICING AND HEDGING IN INCOMPLETE MARKETS WITH COHERENT RISK
}

\author{
Alexander S. Cherny*, Dilip B. Madan ${ }^{* *}$ \\ * Moscow State University \\ Faculty of Mechanics and Mathematics \\ Department of Probability Theory \\ 119992 Moscow, Russia \\ E-mail: cherny@mech.math.msu.su \\ Webpage: http://mech.math.msu.su/ ${ }^{\sim}$ cherny \\ ${ }^{* *}$ Robert H. Smith School of Business \\ Van Munching Hall \\ University of Maryland \\ College Park, MD 20742 \\ E-mail: dmadan@rhsmith.umd.edu \\ Webpage: http://www.rhsmith.umd.edu/faculty/dmadan
}

\begin{abstract}
We propose a pricing technique based on coherent risk measures, which enables one to get finer price intervals than in the No Good Deals pricing. The main idea consists in splitting a liability into several parts and selling these parts to different agents. The technique is closely connected with the convolution of coherent risk measures and equilibrium considerations.

Furthermore, we propose a way to apply the above technique to the coherent estimation of the Greeks.

Key words and phrases. CDO, coherent risk measure, convolution-based pricing, extreme measure, incomplete markets, factor risk, maximum-based pricing, No Strictly Acceptable Opportunities, risk contribution, sensitivity coefficients, valuation measure, Weighted V@R.
\end{abstract}

\section{Contents}

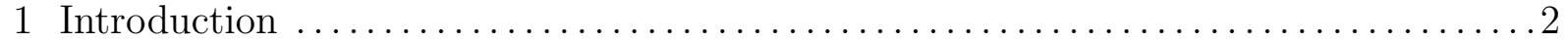

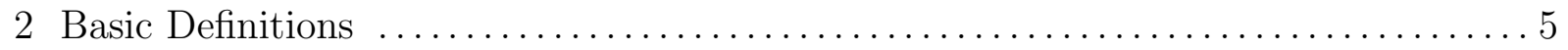

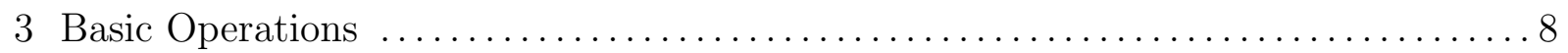

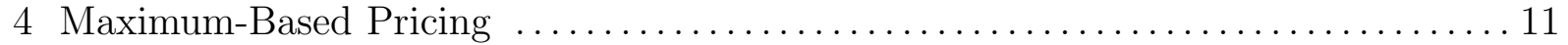

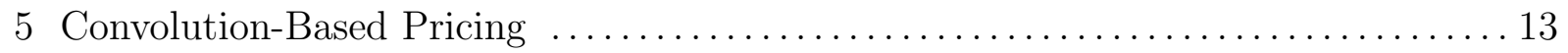

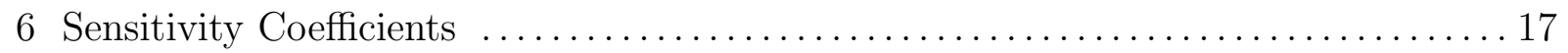

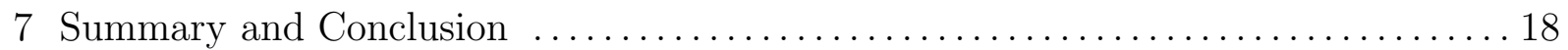

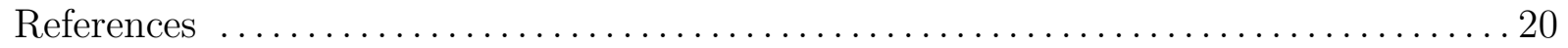




\section{Introduction}

1. Pricing and hedging in incomplete markets. One of the main problems of the modern finance is: How to price derivatives in incomplete markets?

The arbitrage theory typically provides price intervals that are unacceptably large. In order to narrow these intervals, one should use ideas beyond No Arbitrage.

One of possible methods has been proposed by Carr, Geman, and Madan [9]. Their idea is as follows. Suppose that we have $N$ groups of agents and the $n$-th group assesses the quality of any possible trade by taking the expectation of its $\mathrm{P} \& \mathrm{~L} X$ with respect to valuation measures $\mathrm{Q}^{n k}, k=1, \ldots, K^{n}$. Thus, a trade is profitable for this group if $\mathrm{E}_{\mathrm{Q}^{n k}} X>0$ for any $k$. Natural examples of $\mathrm{Q}^{n k}$ are given in Section 3, A trade is called strictly acceptable if its $\mathrm{P} \& \mathrm{~L}$ satisfies $\mathrm{E}_{\mathrm{Q}^{n k}} X>0$ for any $n, k$. The No Strictly Acceptable Opportunities assumption says that such trades do not exist. (This is a natural strengthening of the No Arbitrage assumption.) The fundamental theorem of asset pricing provided in [9] states that strictly acceptable opportunities do not exist if and only if

$$
\left(\underset{n, k}{\operatorname{conv}} \mathrm{Q}^{n k}\right) \cap \mathcal{R} \neq \emptyset
$$

where $\mathcal{R}$ is the set of risk-neutral measures. Using this ideology, one can define the upper and lower prices of a contingent claim $F$ as

$$
\begin{aligned}
& \bar{V}(F)=\inf \left\{x: \exists X \in A \text { such that } \inf _{n, k} \mathrm{E}_{\mathrm{Q}^{n k}}(X-F+x)>0\right\}, \\
& \underline{V}(F)=\sup \left\{x: \exists X \in A \text { such that } \inf _{n, k} \mathrm{E}_{\mathrm{Q}^{n k}}(X+F-x)>0\right\},
\end{aligned}
$$

where $A$ is the set of P\&Ls that can be obtained from various trades available on the market. It follows from the above result that

$$
\begin{aligned}
& \bar{V}(F)=\sup \left\{\mathrm{E}_{\mathrm{Q}} F: \mathrm{Q} \in\left(\underset{n, k}{\operatorname{conv}} \mathrm{Q}^{n k}\right) \cap \mathcal{R}\right\}, \\
& \underline{V}(F)=\inf \left\{\mathrm{E}_{\mathrm{Q}} F: \mathrm{Q} \in\left(\operatorname{conv}_{n, k} \mathrm{Q}^{n k}\right) \cap \mathcal{R}\right\} .
\end{aligned}
$$

Let us remark that this pricing technique is closely connected with the No Good Deals pricing; see [8], 10], 11], 12], [16, 20].

The basic idea of this paper is as follows. Let us call a trade producing a $\mathrm{P} \& \mathrm{~L} X$ strictly acceptable for the $n$-th group if $\mathrm{E}_{\mathrm{Q}^{n k}} X>0$ for any $k=1, \ldots, K^{n}$. Let us now call a trade $X$ strictly acceptable if $X$ can be represented as $X^{1}+\cdots+X^{N}$, where $X^{n}$ is

strictly acceptable for the $n$-th group. The fundamental theorem of asset pricing provided in this paper states that there are no strictly acceptable opportunities if and only if

$$
\left(\bigcap_{n=1}^{N} \operatorname{conv}_{k=1, \ldots, K^{n}} \mathrm{Q}^{n k}\right) \cap \mathcal{R} \neq \emptyset .
$$

Using this ideology, we can define the upper and lower prices of a contingent claim $F$ as

$$
\begin{aligned}
& \bar{V}(F)=\inf \left\{x: \exists X \in A, Y^{1}, \ldots, Y^{N} \text { such that } \sum_{n} Y^{n}=X-F+x\right. \\
& \left.\quad \text { and } \inf _{k} \mathrm{E}_{\mathrm{Q}^{n k}} Y^{n}>0 \text { for any } n\right\}, \\
& \underline{V}(F)=\sup \left\{x: \exists X \in A, Y^{1}, \ldots, Y^{N} \text { such that } \sum_{n} Y^{n}=X+F-x\right. \\
& \left.\quad \text { and } \inf _{k} \mathrm{E}_{\mathrm{Q}^{n k}} Y^{n}>0 \text { for any } n\right\} .
\end{aligned}
$$

In other words, superreplicating a liability consists of two steps: 
1. Trading in the market.

2. Splitting a liability into several parts and selling these parts to different groups at the price 0 .

As shown in the paper,

$$
\begin{aligned}
& \bar{V}(F)=\sup \left\{\mathrm{E}_{\mathrm{Q}} F: \mathrm{Q} \in\left(\bigcap_{n=1}^{N} \operatorname{conv}_{k=1, \ldots, K^{n}} \mathrm{Q}^{n k}\right) \cap \mathcal{R}\right\}, \\
& \underline{V}(F)=\inf \left\{\mathrm{E}_{\mathrm{Q}} F: \mathrm{Q} \in\left(\bigcap_{n=1}^{N} \operatorname{conv}_{k=1, \ldots, K^{n}} \mathrm{Q}^{n k}\right) \cap \mathcal{R}\right\} .
\end{aligned}
$$

Clearly, this technique leads to finer price intervals than the technique described above. A remarkable property is: the more groups are taken into account, the smaller are the fair price intervals. Note that the technique described above has exactly the opposite effect.

Let us remark that the proposed pricing technique is closely connected with the risk sharing (or equilibrium) problem considered in [6], [7, [13, 21].

2. Sensitivity coefficients. Two main goals of asset pricing are:

1. finding fair prices of OTC derivatives;

2. finding sensitivity coefficients, which is needed for risk management.

In the above discussion, we have concentrated on the first problem. Let us now discuss the second one.

Traditionally, in complete models the fair price of a contingent claim is a single number, so that the sensitivity coefficient of the price to the value of an underlying asset can be defined simply by taking the partial derivative. In incomplete markets, we typically have a whole interval of fair prices. The above efforts were aimed at narrowing this interval, but typically it is not a one-point set. Thus, the partial derivative cannot be taken and additional ideas should be used to define sensitivity coefficients. One way to do this was proposed in [16, Subsect. 3.3]. In this paper, we take another path and adjust the pricing technique described above to obtain an interval for a sensitivity.

3. Coherent risk. The pricing technique described above is closely connected with the notion of a coherent risk measure. This notion was introduced by Artzner, Delbaen, Eber, and Heath 4], [5] as a substitute for V@R, which is notorious for its drawbacks. This notion turned out to be extremely useful not only for the risk measurement purposes, but also for other financial problems like pricing and optimization (see [12, 13 and references therein). A coherent risk measure is a function on random variables defined as

$$
\rho(X)=-\inf _{\mathrm{Q} \in \mathcal{D}} \mathrm{E}_{\mathrm{Q}} X,
$$

where $\mathcal{D}$ is a set of probability measures ( $X$ means the P\&L of some transaction). This formula has a clear interpretation: we have a family $\mathcal{D}$ of probabilistic scenarios; we calculate the average P\&L under each scenario; then we take the worst case.

The relationship between the above considerations and coherent risk measures is obvious. Define the coherent risk measure of the $n$-th group as

$$
\rho^{n}(X)=-\inf _{k=1, \ldots, K^{n}} \mathrm{E}_{\mathrm{Q}^{n k}} X .
$$

The first pricing technique says that $X$ is strictly acceptable if $\max _{n} \rho^{n}(X)<0$. For this reason, we call here this technique the maximum-based pricing. Note that $\max _{n} \rho^{n}$ is again a coherent risk measure. 
The second technique says that $X$ is strictly acceptable if $\rho(X):=\min \sum_{n} \rho^{n}\left(X^{n}\right)<0$, where the minimum is taken over all $X^{1}, \ldots, X^{N}$ such that $\sum_{n} X^{n}=X$. The function $\rho$ is again a coherent risk measure termed the convolution of $\rho^{1}, \ldots, \rho^{N}$. For this reason, we call this pricing technique the convolution-based pricing.

Both pricing techniques described above are taking the path

$$
\mathrm{Q}^{n k} \longrightarrow \rho^{n} .
$$

However, one of the most natural ways of constructing the valuation measures $Q^{n k}$ is inverting this arrow, i.e. we start from a coherent risk measure $\rho^{n}$ of the $n$-th group, find the set $\mathcal{D}^{n}$ such that $\rho^{n}(X)=-\inf _{Q \in \mathcal{D}^{n}} X$, and take $\mathcal{D}^{n}$ as the set of valuation measures for the $n$-th group. This is meaningful because typically coherent risk measures are defined not through (1.1), but in a more direct way. For example, the best (in our opinion) one-parameter family of coherent risk measures termed Alpha V@R is defined as:

$$
\rho_{\alpha}(X)=-\mathrm{E} \min _{i=1, \ldots, \alpha} X_{i},
$$

where $\alpha$ is a natural number and $X_{1}, \ldots, X_{\alpha}$ are independent copies of $X$. This class of risk measures was introduced in [15]. As shown in that paper, it is very convenient for the risk measurement purposes.

The sets of valuation measures might be constructed not only from the "pure" risk measures like Alpha V@R, but also through some "derivative" risk measures like factor risks or risk contributions. They might also be obtained through some basic operations on coherent risks.

In typical cases, the sets $\mathcal{D}^{n}$ thus obtained are infinite. Therefore, in this paper we are dealing from the outset with general sets $\mathcal{D}^{n}$ rather than with valuation measures $\mathrm{Q}^{n k}$. Moreover, as opposed to the majority of papers on coherent risk, we are dealing with unbounded random variables (clearly, this is necessary for financial applications) using the framework introduced in [12], 13].

4. Structure of the paper. In Section 2, we recall basic definitions related to coherent risk measures and give several basic examples.

Section 3 has two main goals. First, we provide natural examples of valuation measures. These are based on various transformations of coherent risk measures like factor risks or risk contributions. Second, we describe two basic operations on coherent risks: maximum and convolution. The first operation is at the basis of the pricing technique of Section 4 the second one is at the basis of the pricing technique of Section 5 ,

In Section 4, we consider the maximum-based pricing. This is, in fact, the pricing technique proposed in [9] and extended to a more general framework in [12]. This section is included just for the reader's convenience.

The main results of this paper are presented in Section 5 For the convolution-based pricing technique, we establish the fundamental theorem of asset pricing and find the form of the price intervals. On the mathematical side, our statements easily follow from the results of [12, 13]. We illustrate this technique with an example of calculating the prices and the superreplication strategy for the case, where different groups are using coherent risk measures from the class Weighted V@R. The quantitative effect arising in this example is very similar to how CDOs are arranged. We also discuss the empirical estimation of fair price intervals provided by other natural classes of valuation measures. Furthermore, we study the liquidity effects following the ideas of [9] and the technique of [13]. 
In Section 6, we adjust the convolution-based pricing technique to the assessment of sensitivity coefficients.

\section{Basic Definitions}

1. Coherent risk measures. Let $(\Omega, \mathcal{F}, \mathrm{P})$ be a probability space. Recall that $L^{\infty}$ is the space of bounded random variables on $(\Omega, \mathcal{F}, \mathrm{P})$. The following definition was introduced in [4], [5], 17].

Definition 2.1. A coherent risk measure on $L^{\infty}$ is a map $\rho: L^{\infty} \rightarrow \mathbb{R}$ satisfying the properties:

(a) (Subadditivity) $\rho(X+Y) \leq \rho(X)+\rho(Y)$;

(b) (Monotonicity) If $X \leq Y$, then $\rho(X) \geq \rho(Y)$;

(c) (Positive homogeneity) $\rho(\lambda X)=\lambda \rho(X)$ for $\lambda \in \mathbb{R}_{+}$;

(d) (Translation invariance) $\rho(X+m)=\rho(X)-m$ for $m \in \mathbb{R}$;

(e) (Fatou property) If $\left|X_{n}\right| \leq 1, X_{n} \stackrel{\mathrm{P}}{\rightarrow} X$, then $\rho(X) \leq \liminf _{n} \rho\left(X_{n}\right)$.

The theorem below is the basic representation theorem. It was established in $[5]$ for the case of a finite $\Omega$ (in this case the axiom (e) is not needed) and in [17] for the general case. By $\mathcal{P}$ we will denote the set of probability measures that are absolutely continuous with respect to $P$. Throughout the paper, we identify measures from $\mathcal{P}$ (these are typically denoted by $\mathrm{Q}$ ) with their densities with respect to $\mathrm{P}$ (these are typically denoted by $Z$ ).

Theorem 2.2. A function $\rho$ satisfies conditions (a)-(e) if and only if there exists a non-empty set $\mathcal{D} \subseteq \mathcal{P}$ such that

$$
\rho(X)=-\inf _{\mathbf{Q} \in \mathcal{D}} \mathrm{E}_{\mathbf{Q}} X, \quad X \in L^{\infty} .
$$

Important remark. Suppose that an agent is assessing risk with a coherent risk measure $\rho(X)=-\inf _{\mathrm{Q} \in \mathcal{D}} \mathrm{E}_{\mathrm{Q}} X$. The corresponding risk-adjusted performance is naturally defined as $p(X):=\mathrm{E} X-\lambda \rho(X)$, where $\lambda$ is a positive parameter. Then

$$
(1+\lambda)^{-1} p(X)=\inf _{\mathbf{Q} \in \mathcal{D}^{\prime}} \mathrm{E}_{\mathrm{Q}} X
$$

where

$$
\mathcal{D}^{\prime}=\frac{1}{1+\lambda} \mathrm{P}+\frac{\lambda}{1+\lambda} \mathcal{D} \text {. }
$$

The negative of a coherent risk measure is called a coherent utility function (in some respects this object is more convenient than a coherent risk measure because it eliminates numerous minus signs). Thus, $(1+\lambda)^{-1} p(X)$ is a coherent utility. This stability property shows that coherent risk measures can be used not only to measure risk, but to measure risk-adjusted performance, i.e. utility as well.

Throughout this paper, we deal with coherent risk measures on the space $L^{0}$ of all random variables. The following definition was introduced in [12].

Definition 2.3. A coherent risk measure on $L^{0}$ is a map $\rho: L^{0} \rightarrow[-\infty, \infty]$ defined as

$$
\rho(X)=-\inf _{\mathrm{Q} \in \mathcal{D}} \mathrm{E}_{\mathrm{Q}} X, \quad X \in L^{0}
$$

where $\mathcal{D}$ is a non-empty subset of $\mathcal{P}$ and $\mathrm{E}_{\mathrm{Q}} X$ is understood as $\mathrm{E}_{\mathrm{Q}} X^{+}-\mathrm{E}_{\mathrm{Q}} X^{-}$ $\left(X^{+}=\max \{X, 0\}, X^{-}=\max \{-X, 0\}\right)$ with the convention $\infty-\infty=-\infty$. 
The set $\mathcal{D}$, for which (2.1) is true, is not unique. However, there exists the largest such set. It is given by $\left\{\mathrm{Q} \in \mathcal{P}: \mathrm{E}_{\mathrm{Q}} X \geq-\rho(X)\right.$ for any $\left.X\right\}$.

Definition 2.4. The largest set, for which (2.1) is true, is called the determining set of $\rho$.

Important remark. Let $\mathcal{D}$ be a subset of $\mathcal{P}$. Define a coherent risk measure $\rho$ by (2.1). The determining set of $\rho$ might be strictly larger than $\mathcal{D}$. However, if $\mathcal{D}$ is convex and $L^{1}$-closed, then $\mathcal{D}$ is the determining set of $\rho$. Indeed, suppose that the determining set $\widetilde{\mathcal{D}}$ is larger than $\mathcal{D}$. Choose $Q_{0} \in \widetilde{\mathcal{D}} \backslash \mathcal{D}$. By the Hahn-Banach theorem, there exists $X \in L^{\infty}$ such that $\mathrm{E}_{\mathrm{Q}_{0}} X<\inf _{\mathrm{Q} \in \mathcal{D}} \mathrm{E}_{\mathrm{Q}} X=-\rho(X)$, which is a contradiction.

For more information on coherent risk measures, we refer to [15], [18, and [19, Sect. 4].

2. Examples. Let us give examples of four most natural classes of coherent risk measures.

Example 2.5 (Tail V@R). Tail V@R of order $\lambda \in(0,1]$ (the terms Average V@R, ConditionalV@R, Expected Shortfall, and Expected Tail Loss are also used) is the coherent risk measure $\rho_{\lambda}$ corresponding to the determining set

$$
\mathcal{D}_{\lambda}=\left\{\mathrm{Q} \in \mathcal{P}: \frac{d \mathrm{Q}}{d \mathrm{P}} \leq \lambda^{-1}\right\}
$$

If $X$ has a continuous distribution, then

$$
\rho_{\lambda}(X)=-\mathrm{E}\left(X \mid X \leq q_{\lambda}(X)\right),
$$

where $q_{\lambda}(X)$ is the $\lambda$-quantile of $X$. This motivates the term Tail V@R.

For a detailed study of this risk measure, we refer to [3], [15, Sect. 2], [19; Sect. 4.4].

Example 2.6 (Weighted V@R). Let $\mu$ be a probability measure on $(0,1]$. Weighted $V @ R$ with the weighting measure $\mu$ (the term spectral risk measure is also used) is the coherent risk measure $\rho_{\mu}$ defined as

$$
\rho_{\mu}(X)=\int_{(0,1]} \rho_{\lambda}(X) \mu(d \lambda) .
$$

(One can check that this is indeed a coherent risk measure.)

Weighted V@R admits several equivalent representations. One of the most convenient representations is:

$$
\rho_{\mu}(X)=-\int_{0}^{1} q_{x}(X) \psi_{\mu}(x) d x
$$

where

$$
\psi_{\mu}(x)=\int_{[x, 1]} \lambda^{-1} \mu(d \lambda), \quad x \in[0,1] .
$$

In particular, suppose that $X$ takes on values $x_{1}, \ldots, x_{T}$ with probabilities $p_{1}, \ldots, p_{T}$. Let $x_{(1)}, \ldots, x_{(T)}$ be the numbers $x_{1}, \ldots, x_{T}$ in the increasing order and let $n(i)$ be the number such that $x_{(i)}=x_{n(i)}$. Then

$$
\rho_{\mu}(X)=-\sum_{t=1}^{T} x_{n(t)} \int_{z_{t-1}}^{z_{t}} \psi_{\mu}(x) d x,
$$


where $z_{t}=\sum_{i=1}^{t} p_{n(i)}$. This representation is convenient for the empirical estimation of $\rho$. The determining set $\mathcal{D}_{\mu}$ of $\rho_{\mu}$ admits the following representations:

$$
\begin{aligned}
\mathcal{D}_{\mu} & =\left\{\mathrm{Q} \in \mathcal{P}: \mathrm{Q}(A) \leq \Psi_{\mu}(\mathrm{P}(A)) \text { for any } A \in \mathcal{F}\right\} \\
& =\left\{Z \in L^{0}: Z \geq 0, \mathrm{E}_{\mathrm{P}} Z=1, \text { and } \int_{1-x}^{1} q_{s}(Z) d s \leq \Psi_{\mu}(x) \forall x \in[0,1]\right\} \\
& =\left\{Z \in L^{0}: Z \geq 0, \mathrm{E} Z=1, \text { and } \mathrm{E}(Z-x)^{+} \leq \Phi_{\mu}(x) \forall x \in \mathbb{R}_{+}\right\},
\end{aligned}
$$

where

$$
\begin{aligned}
& \Psi_{\mu}(x)=\int_{0}^{x} \psi_{\mu}(y) d y, \quad x \in[0,1], \\
& \Phi_{\mu}(x)=\sup _{y \in[0,1]}\left(\Psi_{\mu}(y)-x y\right), \quad x \in \mathbb{R}_{+}
\end{aligned}
$$

(see 14, Th. 4.6], 19, Th. 4.73], 23, Th. 1.53]).

For a detailed study of Weighted V@R, we refer to [1, [2, [14, [15, Sect. 2], 19, Sect. 4.6, 4.7].

Example 2.7 (Beta V@R). Let $\alpha \in(-1, \infty), \beta \in(-1, \alpha)$. Beta $V @ R$ with parameters $\alpha, \beta$ is the Weighted $\mathrm{V} @ \mathrm{R}$ with the weighting measure

$$
\mu_{\alpha, \beta}(d x)=\mathrm{B}(\beta+1, \alpha-\beta)^{-1} x^{\beta}(1-x)^{\alpha-\beta-1} d x, \quad x \in[0,1] .
$$

As shown in [15], for $\alpha, \beta \in \mathbb{N}$, Beta V@R admits the following simple representation

$$
\rho_{\alpha, \beta}(X)=-\mathrm{E}\left[\frac{1}{\beta} \sum_{i=1}^{\beta} X_{(i)}\right],
$$

where $X_{(1)}, \ldots, X_{(\alpha)}$ are the order statistics obtained from independent copies $X_{1}, \ldots, X_{\alpha}$ of $X$. This representation provides a very convenient way for the empirical estimation of $\rho_{\alpha, \beta}$.

For a detailed study of this risk measure, see [15; Sect. 2].

Example 2.8 (Alpha V@R). Alpha $V @ R$ is obtained from Beta V@R by fixing $\beta=1$. Clearly, if $\alpha \in \mathbb{N}$, then

$$
\rho_{\alpha}(X)=-\mathrm{E} \min _{i=1, \ldots, \alpha} X_{i},
$$

where $X_{1}, \ldots, X_{\alpha}$ are independent copies of $X$.

In our opinion, the most important classes of coherent risk measures are: Alpha V@R, Beta V@R, and Weighted V@R.

3. $L^{1}$-spaces. For technical purposes, we need to recall the definition of the strong $L^{1}$-space associated with a coherent risk measure $\rho$ :

$$
L_{s}^{1}(\mathcal{D})=\left\{X \in L^{0}: \lim _{n \rightarrow \infty} \sup _{\mathbf{Q} \in \mathcal{D}} \mathrm{E}_{\mathrm{Q}}|X| I(|X|>n)=0\right\}
$$

where $\mathcal{D}$ is the determining set of $\rho$.

Example 2.9. (i) For Weighted V@R,

$$
L_{s}^{1}\left(\mathcal{D}_{\mu}\right)=\left\{X \in L^{0}: \rho(X)<\infty, \rho(-X)<\infty\right\}
$$

(see [12, Subsect. 2.2]). The right-hand side of this equality was called in [12] the weak $L^{1}$-space. It has a clear financial interpretation: this is the set of random variables such that their risk is finite and the risk of their negatives is finite.

(ii) For Beta V@R with $\beta>0$ (in particular, for Alpha V@R), $L_{s}^{1}=L^{1}(\mathrm{P})$ (see [15. Sect. 3]). 


\section{Basic Operations}

1. Factor risks. Suppose that an agent is assessing risk using a coherent risk measure $\rho$. As proposed in [15], it is important to consider not only the pure risk $\rho(X)$, but also the factor risks

$$
\rho^{f}\left(X ; Y^{m}\right)=-\inf _{\mathbf{Q} \in \mathrm{E}\left(\mathcal{D} \mid Y^{m}\right)} \mathrm{E}_{\mathrm{Q}} X, \quad m=1, \ldots, M .
$$

Here $Y^{1}, \ldots, Y^{M}$ are the main market factors affecting risk like the price of oil, the S\&P 500 index, or the credit spread (to be more precise, $Y^{m}$ is the increment of the $m$-th factor over the unit time period), $X$ means the $\mathrm{P} \& \mathrm{~L}$ produced by some portfolio over this period, and

$$
\mathrm{E}\left(\mathcal{D} \mid Y^{m}\right):=\{\mathrm{E}(Z \mid Y): Z \in \mathcal{D}\}
$$

where $\mathcal{D}$ is the determining set of $\rho$. Thus, $\rho^{f}\left(\cdot ; Y^{m}\right)$ is again a coherent risk measure with the determining set $\mathrm{E}\left(\mathcal{D} \mid Y^{m}\right)$. As shown in [15], under minor technical assumptions,

$$
\rho^{f}\left(X ; Y^{m}\right)=\rho\left(\mathrm{E}\left(X \mid Y^{m}\right)\right) .
$$

If an agent is using these measures to assess risk, he/she might take $\operatorname{conv}_{m} \mathrm{E}\left(\mathcal{D} \mid Y^{m}\right)$ as the set of his/her valuation measures.

Another opportunity is that the agent is assessing risk using one multi-factor risk measure

$$
\rho^{f}\left(X ; Y^{1}, \ldots, Y^{M}\right):=-\inf _{\mathbf{Q} \in \mathrm{E}\left(\mathcal{D} \mid Y^{1}, \ldots, Y^{M}\right)} \mathrm{E}_{\mathrm{Q}} X=\rho\left(\mathrm{E}\left(X \mid Y^{1}, \ldots, Y^{M}\right)\right) .
$$

Then he/she might take $\mathrm{E}\left(\mathcal{D} \mid Y^{1}, \ldots, Y^{M}\right)$ as the set of his/her valuation measures.

2. Risk contribution. Measurement of pure risk/utility is meaningful only for a "poor" agent, i.e. an agent without a large endowment. A wealthy agent (for example, a big company) already has a large portfolio that produces a random P\&L $W$. For such an agent, it is reasonable to assess any trade $X$ as $\rho(W+X)-\rho(W)$ rather than as $\rho(X)$. In other words, the quantity of interest is the risk contribution of $X$ to $W$. According to the definition introduced in [12, the risk contribution is

$$
\rho^{c}(X ; W)=-\inf _{\mathbf{Q} \in \mathcal{X}_{\mathcal{D}}(W)} \mathrm{E}_{\mathrm{Q}} X
$$

where $\mathcal{X}_{\mathcal{D}}(W)$ is the set of extreme measures defined as

$$
\mathcal{X}_{\mathcal{D}}(W)=\left\{\mathrm{Q} \in \mathcal{D}: \mathrm{E}_{\mathrm{Q}} W=\inf _{\mathbf{Q} \in \mathcal{D}} \mathrm{E}_{\mathrm{Q}} W \in(-\infty, \infty)\right\} .
$$

The relevance of this definition is justified by the following relation (see [12; Subsect. 2.5]):

$$
\rho^{c}(X ; W)=\lim _{\varepsilon \downarrow 0} \varepsilon^{-1}(\rho(W+\varepsilon X)-\rho(W)) .
$$

Thus, $\mathcal{X}_{\mathcal{D}}(W)$ is a natural candidate for the set of valuation measures for a wealthy agent employing coherent risk.

If an agent is using the classical expected utility $\mathrm{E} U(X)$ to assess the quality of his/her position (here $U: \mathbb{R} \rightarrow \mathbb{R}$ is a concave increasing function), then there exists his/her "personal" measure, with which he/she assesses the quality of any possible trade. 
This measure is given by $\mathrm{Q}=c U^{\prime}\left(W_{1}\right) \mathrm{P}$, where $W_{1}$ is the agent's wealth at the terminal date and $c$ is the normalizing constant. The role of this measure is seen from the equality

$$
\lim _{\varepsilon \downarrow 0} \varepsilon^{-1}\left(\mathrm{E} U\left(W_{1}+\varepsilon X\right)-\mathrm{E} U\left(W_{1}\right)\right)=\mathrm{E} X U^{\prime}\left(W_{1}\right)=c^{-1} \mathrm{E}_{\mathrm{Q}} X .
$$

If $X$ is the P\&L produced by some trade and $X$ is small as compared to $W$, then $X$ is profitable for the agent if and only if $\mathrm{E}_{\mathrm{Q}} X>0$.

Thus, the notion of an extreme measure serves as the coherent counterpart of $c U^{\prime}\left(W_{1}\right) \mathrm{P}$. The set $\mathcal{X}_{\mathcal{D}}(W)$ is typically a singleton as seen from the examples below.

Example 3.1. (i) If $W \in L_{s}^{1}\left(\mathcal{D}_{\mu}\right)$ has a continuous distribution, then $\mathcal{X}_{\mathcal{D}_{\mu}}(W)$ consists of a unique measure $\mathrm{Q}_{\mu}(W)=\psi_{\mu}(F(W)) \mathrm{P}$, where $\psi_{\mu}$ is given by (2.3) and $F$ is the distribution function of $W$ (for the proof, see [14, Sect. 6]).

(ii) Let $\Omega=\{1, \ldots, T\}$ and $W(t)=w_{t}$, where all $w_{n}$ are different. Let $w_{(1)}, \ldots, w_{(T)}$ be the numbers $w_{1}, \ldots, w_{T}$ in the increasing order and let $n(i)$ be the number such that $w_{(i)}=w_{n(i)}$. Then $\mathcal{X}_{\mathcal{D}_{\mu}}(W)$ consists of a unique measure $\mathbf{Q}_{\mu}(W)$ given by

$$
\mathrm{Q}_{\mu}(W)\{n(t)\}=\int_{z_{t-1}}^{z_{t}} \psi_{\mu}(x) d x
$$

where $\psi_{\mu}$ is given by (2.3) and $z_{t}=\sum_{i=1}^{t} \mathrm{P}\{n(i)\}$ (for the proof, see [15, Sect. 5]).

3. Factor risk contribution. The risk contribution technique can be combined with the factor risk technique. Namely, the factor risk contribution is defined as

$$
\rho^{f c}(X ; Y ; W)=-\inf _{\mathbf{Q} \in \mathcal{X}_{\mathbb{E}(\mathcal{D} \mid Y)}(W)} \mathrm{E}_{Q} X .
$$

Here $Y$ means the increment of one or several market factors over the unit time period. As shown in [15], under minor technical assumptions,

$$
\rho^{f c}(X ; Y ; W)=\rho^{c}(\mathrm{E}(X \mid Y) ; \mathrm{E}(W \mid Y)) .
$$

Thus, $\mathcal{X}_{\mathrm{E}(\mathcal{D} \mid Y)}(W)$ is a natural candidate for the set of valuation measures for a wealthy agent employing factor risk.

Example 3.2. Combining representation (2.5) with the Jensen inequality, we see that

$$
\begin{aligned}
\mathrm{E}\left(\mathcal{D}_{\mu} \mid Y\right)= & \left\{\varphi(Y): \varphi \geq 0, \int_{\mathbb{R}^{d}} \varphi(x) \widetilde{\mathrm{P}}(d x)=1,\right. \text { and } \\
& \left.\int_{\mathbb{R}^{d}}(\varphi(x)-K)^{+} \widetilde{\mathrm{P}}(d x) \leq \Phi_{\mu}(K) \forall K \in \mathbb{R}_{+}\right\} \\
= & \left\{\varphi(Y): \varphi \in \widetilde{\mathcal{D}}_{\mu}\right\},
\end{aligned}
$$

where $\widetilde{\mathrm{P}}=$ Law $Y$ ( $Y$ is $d$-dimensional $)$ and $\widetilde{\mathcal{D}}_{\mu}$ is the determining set of Weighted V@R defined on the space $(\widetilde{\Omega}, \widetilde{\mathcal{F}}, \widetilde{\mathrm{P}})=\left(\mathbb{R}^{d}, \mathcal{B}, \widetilde{\mathrm{P}}\right)$. Let $W \in L_{s}^{1}\left(\mathcal{D}_{\mu}\right)$. Set $g(y)=\mathrm{E}(W \mid Y=y)$ (recall from Example 2.9 that $\left.L_{s}^{1}\left(\mathcal{D}_{\mu}\right) \subseteq L^{1}\right)$. Then

$$
\mathrm{E} \varphi(Y) W=\mathrm{E} \varphi(Y) g(Y)=\int_{\mathbb{R}^{d}} \varphi(y) g(y) \widetilde{\mathrm{P}}(d y), \quad \varphi \in \widetilde{\mathcal{D}}_{\mu},
$$

so that

$$
\mathcal{X}_{\mathrm{E}\left(\mathcal{D}_{\mu} \mid Y\right)}(W)=\left\{\varphi(Y): \varphi \in \widetilde{\mathcal{X}}_{\widetilde{\mathcal{D}}_{\mu}}(g)\right\} .
$$


For more information on factor risks and risk contributions, we refer to [12], [15].

4. Maximum of coherent risks. Let $\rho^{1}, \ldots, \rho^{N}$ be coherent risk measures with the determining sets $\mathcal{D}^{1}, \ldots, \mathcal{D}^{N}$. We assume that each $\mathcal{D}^{n}$ is $L^{1}$-closed and uniformly integrable. This assumption is very natural: as seen from (2.5), it is satisfied by the determining set of Weighted V@R. If $\mathcal{D}$ satisfies this assumption, then $\mathrm{E}(\mathcal{D} \mid Y)$ also has this property (for the proof, see [12, Sect. 3]). It is easy to check that if $\mathcal{D}$ satisfies this assumption and $W \in L_{s}^{1}(\mathcal{D})$, then $\mathcal{X}_{\mathcal{D}}(W)$ also has this property.

The maximum of $\rho^{1}, \ldots, \rho^{N}$ is defined as

$$
\rho(X)=-\inf _{\mathbf{Q} \in \operatorname{conv}_{n} \mathcal{D}^{n}} \mathrm{E}_{\mathrm{Q}} X .
$$

According to the remark following Definition 2.4 $\operatorname{conv}_{n} \mathcal{D}^{n}$ is the determining set of $\rho$. Obviously,

$$
\rho(X)=\max _{n=1, \ldots, N} \rho^{n}(X)
$$

which motivates the term.

The operation of taking maximum is useful if an agent has several alternative coherent ways to assess risk and is on the safe side provided that all these risks of his/her position are negative. Another possible interpretation is: we have a group of agents employing different coherent risk measures; then the maximum is the most liberal one.

5. Convolution of coherent risks. Let $\rho^{1}, \ldots, \rho^{N}$ be coherent risk measures with the determining sets $\mathcal{D}^{1}, \ldots, \mathcal{D}^{N}$. We assume that each $\mathcal{D}^{1}$ is closed and uniformly integrable and $\bigcap_{n} \mathcal{D}^{n} \neq \emptyset$.

The convolution of $\rho^{1}, \ldots, \rho^{N}$ is defined as

$$
\rho(X)=-\inf _{\mathbf{Q} \in \bigcap_{n} \mathcal{D}^{n}} \mathrm{E}_{\mathrm{Q}} X
$$

According to the remark following Definition $2.4, \bigcap_{n} \mathcal{D}^{n}$ is the determining set of $\rho$. It follows from [13, Th. 4.2] that, for $X \in L_{s}^{1}:=\bigcap_{n} L_{s}^{1}\left(\mathcal{D}^{n}\right)$,

$$
\rho(X)=\inf _{X^{n} \in L_{s}^{1}, \sum_{n} X^{n}=X} \sum_{n=1}^{N} \rho^{n}\left(X^{n}\right) .
$$

Example 3.3. (i) It is seen from (2.5) that

$$
\bigcap_{n=1}^{N} \mathcal{D}_{\mu^{n}}=\left\{\mathrm{Q} \in \mathcal{P}: \mathrm{Q}(A) \leq \min _{n} \Psi_{\mu^{n}}(\mathrm{P}(A)) \text { for any } A \in \mathcal{F}\right\} .
$$

The function $\Psi=\min _{n} \Psi_{\mu^{n}}$ is increasing, concave, continuous, $\Psi(0)=0$, and $\Psi(1)=1$. Hence, $\Psi=\Psi_{\mu}$ with $\mu(d x)=-x \Psi^{\prime \prime}(d x)$, where $\Psi^{\prime \prime}$ is the second derivative of $\Psi$ taken in the sense of distributions, i.e. it is the measure on $(0,1]$ defined by $\Psi^{\prime \prime}((a, b]):=\Psi_{+}^{\prime}(b)-\Psi_{+}^{\prime}(a)$, where $\Psi_{+}^{\prime}$ is the right-hand derivative. Thus, the convolution of Weighted V@Rs is again a Weighted V@R.

(ii) The maximum of Weighted V@Rs need not be a Weighted V@R as shown by the following example. Consider $\mu^{1}=1 / 2 \delta_{1 / 3}+1 / 2 \delta_{1}, \mu^{2}=\delta_{2 / 3}$. We have

$$
\Psi_{\mu^{1}}(x)=\left\{\begin{array}{ll}
2 x, & 0 \leq x \leq \frac{1}{3}, \\
\frac{1}{2} x+\frac{1}{2}, & \frac{1}{3} \leq x \leq 1,
\end{array} \quad \Psi_{\mu^{2}}(x)= \begin{cases}\frac{3}{2} x, & 0 \leq x \leq \frac{2}{3} \\
1, & \frac{2}{3} \leq x \leq 1\end{cases}\right.
$$




\begin{tabular}{|l|l|l|}
\hline \multicolumn{1}{|c|}{$\begin{array}{c}\text { Risk/utility } \\
\text { measurement technique }\end{array}$} & \multicolumn{1}{c|}{ Inputs } & \multicolumn{1}{c|}{$\begin{array}{c}\text { Valuation } \\
\text { measures }\end{array}$} \\
\hline \hline Coherent risk/utility & $\rho$ & $\mathcal{D}$ \\
\hline Factor risk/utility & $\rho, Y^{1}, \ldots, Y^{M}$ & $\operatorname{conv}_{m} \mathrm{E}\left(\mathcal{D} \mid Y^{m}\right)$ \\
\hline Multi-factor risk/utility & $\rho, Y^{1}, \ldots, Y^{M}$ & $\mathrm{E}\left(\mathcal{D} \mid Y^{1}, \ldots, Y^{M}\right)$ \\
\hline Risk/utility contribution & $\rho, W$ & $\mathcal{X}_{\mathcal{D}}(W)$ \\
\hline Factor risk/utility contribution & $\rho, Y^{1}, \ldots, Y^{M}, W$ & $\operatorname{conv}_{m} \mathcal{X}_{\mathrm{E}\left(\mathcal{D} \mid Y^{m}\right)}(W)$ \\
\hline Multi-factor risk/utility contribution & $\rho, Y^{1}, \ldots, Y^{M}, W$ & $\mathcal{X}_{\mathrm{E}\left(\mathcal{D} \mid Y^{1}, \ldots, Y^{M}\right)}(W)$ \\
\hline Expected utility & $U, W_{1}$ & $c U^{\prime}\left(W_{1}\right) \mathrm{P}$ \\
\hline Combination of several techniques & $\mathcal{V}^{1}, \ldots, \mathcal{V}^{K}$ & $\operatorname{conv}_{k} \mathcal{V}^{k}$ \\
\hline
\end{tabular}

Table 1. Construction of valuation measures

Suppose that the maximum of $\rho_{\mu^{1}}, \rho_{\mu^{2}}$ has the form $\rho_{\mu}$ with some $\mu$. As seen from (2.5), $\Psi_{\mu}$ should coincide with the minimal concave majorant of $\Psi_{\mu^{1}}$ and $\Psi_{\mu^{2}}$, i.e.

$$
\Psi_{\mu}(x)= \begin{cases}2 x, & 0 \leq x \leq \frac{1}{3} \\ x+\frac{1}{3}, & \frac{1}{3} \leq x \leq \frac{2}{3} \\ 1, & \frac{2}{3} \leq x \leq 1\end{cases}
$$

This means that $\mu=1 / 3 \delta_{1 / 3}+2 / 3 \delta_{2 / 3}$. Now, by considering a random variable taking on the values $-1,0$, and 1000 with probability $1 / 3$, we see that $\rho_{\mu}$ is not the maximum of $\rho_{\mu^{1}}, \rho_{\mu^{2}}$.

For more information on operations on coherent risk measures, we refer to [18, Sect. 5].

6. Valuation measures. Table 1 summarizes various possible ways of constructing valuation measures.

The first 3 lines correspond to a "poor" agent using one of 3 possible coherent ways of risk measurement. As indicated in the remark following Theorem 2.2, the negatives of coherent risk measures might be used to measure utility.

The next 3 lines correspond to a wealthy agent who measures risk/utility in a coherent way, while the 7 th line corresponds to a wealthy agent employing expected utility.

Finally, the 8th line corresponds to the case, where an agent is employing various risk/ulitity measurement techniques (that yield the sets $\mathcal{V}^{1}, \ldots, \mathcal{V}^{K}$ of valuation measures) or we have a group consisting of several agents, each with his/her own set $\mathcal{V}^{k}$ of valuation measures.

\section{Maximum-Based Pricing}

Let $(\Omega, \mathcal{F}, \mathrm{P})$ be a probability space and $\mathcal{V}^{1}, \ldots, \mathcal{V}^{N}$ be convex $L^{1}$-closed uniformly integrable subsets of $\mathcal{P}$. From the financial point of view, $\mathcal{V}^{n}$ is the set of valuation measures used by the $n$-th group. Let $A$ be a convex subset of $L_{s}^{1}:=\bigcap_{n} L_{s}^{1}\left(\mathcal{V}^{n}\right)$. From the financial point of view, this is the set of various discounted P\&Ls that can be obtained by various 
trading operations over the unit time period. For example, if the agents can trade the assets $1, \ldots, d$ and the $i$-th asset produces the discounted P\&L $X^{i}$, then

$$
A=\left\{\sum_{i=1}^{d} h^{i} X^{i}:\left(h^{1}, \ldots, h^{d}\right) \in H\right\}
$$

where $H$ is a portfolio constraint (if there are no constraints, then $H=\mathbb{R}^{d}$ ).

Definition 4.1. (i) The set of strictly acceptable opportunities for the $n$-th group is

$$
\mathcal{A}^{n}:=\left\{X \in L_{s}^{1}: \inf _{\mathrm{Q} \in \mathcal{V}^{n}} \mathrm{E}_{\mathrm{Q}} X>0\right\}
$$

(ii) The set of strictly acceptable opportunities is

$$
\mathcal{A}=\left\{X \in L_{s}^{1}: X \in \mathcal{A}^{n} \forall n\right\}
$$

Lemma 4.2. Let $\rho^{n}$ be the coherent risk measure with the determining set $\mathcal{V}^{n}$ and let $\rho$ be the maximum of $\rho^{1}, \ldots, \rho^{N}$. Then

$$
\mathcal{A}=\bigcap_{n=1}^{N} \mathcal{A}^{n}=\left\{X \in L_{s}^{1}: \rho(X)<0\right\} .
$$

This statement is trivial.

Definition 4.3. The model satisfies the No Strictly Acceptable Opportunities (NSAO) condition if $A \cap \mathcal{A}=\emptyset$.

Definition 4.4. A risk-neutral measure is a measure $\mathrm{Q} \in \mathcal{P}$ such that $\mathrm{E}_{\mathrm{Q}} X \leq 0$ for any $X \in A$ (the expectation $\mathrm{E}_{\mathrm{Q}} X$ here is understood as $\mathrm{E}_{\mathrm{Q}} X^{+}-\mathrm{E}_{\mathrm{Q}} X^{-}$with the convention $\infty-\infty=-\infty)$.

The set of risk-neutral measures will be denoted by $\mathcal{R}$.

Theorem 4.5 (FTAP). The NSAO condition is satisfied if and only if $\left(\operatorname{conv}_{n} \mathcal{V}^{n}\right) \cap \mathcal{R} \neq \emptyset$.

Proof. By Lemma 4.2, the NSAO condition coincides with the No Good Deals (NGD) condition defined in [12] and applied to the coherent risk measure $\rho$ with the determining set $\mathcal{V}=\operatorname{conv}_{n} \mathcal{V}^{n}$. Now, the result follows from [12; Th. 3.4].

Definition 4.6. Let $F \in L^{0}$ be the discounted payoff of some contingent claim. The upper and lower prices of $F$ are defined as

$$
\begin{aligned}
& \bar{V}(F)=\inf \{x \in \mathbb{R}: \exists X \in A \text { such that } X-F+x \in \mathcal{A}\} \\
& \underline{V}(F)=\sup \{x \in \mathbb{R}: \exists X \in A \text { such that } X+F-x \in \mathcal{A}\} .
\end{aligned}
$$

Theorem 4.7 (Pricing). If $A$ is a cone and $F \in L_{s}^{1}$, then

$$
\begin{aligned}
& \bar{V}(F)=\sup \left\{\mathrm{E}_{\mathrm{Q}} F: \mathrm{Q} \in\left(\operatorname{conv}_{n} \mathcal{V}^{n}\right) \cap \mathcal{R}\right\}, \\
& \underline{V}(F)=\inf \left\{\mathrm{E}_{\mathrm{Q}} F: \mathrm{Q} \in\left(\operatorname{conv}_{n} \mathcal{V}^{n}\right) \cap \mathcal{R}\right\} .
\end{aligned}
$$


Proof. Take $x_{0} \in \mathbb{R}$, set $A\left(x_{0}\right)=A+\left\{h\left(x_{0}-F\right): h \in \mathbb{R}_{+}\right\}$, and denote the corresponding set of risk-neutral measures by $\mathcal{R}\left(A\left(x_{0}\right)\right)$. Clearly, the set $\{x: \exists X \in A$ such that $X-F+x \in \mathcal{A}\}$ is an open ray. Using Theorem 4.5, we can write

$$
\begin{aligned}
\bar{V}(F) \geq x_{0} & \Longleftrightarrow \nexists X \in A \text { such that } X-F+x_{0} \in \mathcal{A} \\
& \Longleftrightarrow A\left(x_{0}\right) \cap \mathcal{A}=\emptyset \\
& \Longleftrightarrow\left(\operatorname{conv}_{n} \mathcal{V}^{n}\right) \cap \mathcal{R}\left(A\left(x_{0}\right)\right) \neq \emptyset \\
& \Longleftrightarrow \exists \mathrm{Q} \in\left(\operatorname{conv}_{n} \mathcal{V}^{n}\right) \cap \mathcal{R} \text { such that } \mathrm{E}_{\mathrm{Q}} F \geq x_{0}
\end{aligned}
$$

This yields the formula for $\bar{V}(F)$. The representation of $\underline{V}(F)$ is proved similarly.

Remarks. (i) The above theorem is formally true if the NSAO is violated. In this case $\bar{V}(F)=-\infty$ and $\underline{V}(F)=\infty$.

(ii) The above argument shows that there exist $\bar{Q}, \underline{Q} \in\left(\operatorname{conv}_{n} \mathcal{V}^{n}\right) \cap \mathcal{R}$ such that $\mathrm{E}_{\overline{\mathrm{Q}}} F=\bar{V}(F), \mathrm{E}_{\underline{Q}}(F)=\underline{V}(F)$. This is in contrast with the No Arbitrage technique.

\section{Convolution-Based Pricing}

1. General setup. Let $\mathcal{V}^{n}, A$, and $\mathcal{A}^{n}$ be the same as above.

Definition 5.1. The set of strictly acceptable opportunities is

$$
\mathcal{A}=\left\{X \in L_{s}^{1}: \exists X^{1}, \ldots, X^{N} \in L_{s}^{1} \text { such that } \sum_{n} X^{n}=X \text { and } X^{n} \in \mathcal{A}^{n} \forall n\right\} .
$$

Lemma 5.2. Let $\rho^{n}$ be the coherent risk measure with the determining set $\mathcal{V}^{n}$ and let $\rho$ be the convolution of $\rho^{1}, \ldots, \rho^{N}$. Then

$$
\mathcal{A}=\operatorname{conv}_{n} \mathcal{A}^{n}=\left\{X \in L_{s}^{1}: \rho(X)<0\right\} .
$$

This statement follows from (3.3).

The NSAO condition, the set of risk-neutral measures, and the upper and lower prices are defined similarly as above.

Theorem 5.3 (FTAP). The NSAO condition is satisfied if and only if $\left(\bigcap_{n} \mathcal{V}^{n}\right) \cap \mathcal{R} \neq \emptyset$.

The proof is similar to the proof of Theorem 4.5.

Theorem 5.4 (Pricing). If $A$ is a cone and $F \in L_{s}^{1}$, then

$$
\begin{aligned}
& \bar{V}(F)=\sup \left\{\mathrm{E}_{\mathrm{Q}} F: \mathrm{Q} \in\left(\bigcap_{n} \mathcal{V}^{n}\right) \cap \mathcal{R}\right\}, \\
& \underline{V}(F)=\inf \left\{\mathrm{E}_{\mathrm{Q}} F: \mathrm{Q} \in\left(\bigcap_{n} \mathcal{V}^{n}\right) \cap \mathcal{R}\right\} .
\end{aligned}
$$

The proof is similar to the proof of Theorem 4.7.

In the maximum-based pricing, superreplicating a contingent claim $F$ means applying a trading strategy $X_{*}$ such that $\rho^{n}\left(X_{*}-F+\bar{V}(F)\right) \leq 0$ for any $n$. The motivation is that the liability $X_{*}-F+\bar{V}(F)$ is riskless because it can be sold to any group at the price 0 . For the convolution-based pricing, superreplication is a more complicated procedure consisting of two steps: 
1. Apply a trading strategy $X_{*}$ such that $\rho\left(X_{*}-F+\bar{V}(F)\right)=0$.

2. Split the liability $X_{*}-F+\bar{V}(F)$ into contracts $Y_{*}^{1}, \ldots, Y_{*}^{N}$ such that $\rho^{n}\left(Y_{*}^{n}\right)=0$ for any $n$ and sell the $n$-th contract to the $n$-th group.

To sum up, a superreplication strategy is a collection $X_{*}, Y_{*}^{1}, \ldots, Y_{*}^{N}$.

2. Theoretical example. Let us present the explicit solution of the pricing and hedging problem in the case, where $\mathcal{V}^{n}$ is the determining set of $\rho_{\mu^{n}}$. According to Example 3.3 (i), $\rho=\rho_{\mu}$ with $\mu(d x)=-x \Psi^{\prime \prime}(x)$, where $\Psi=\min _{n} \Psi_{\mu^{n}}$. Therefore,

$$
\bar{V}(F)=-\inf _{X \in A} \rho_{\mu}(X-F) .
$$

Theorem 5.5. (i) Suppose that there exists

$$
X_{*} \in \underset{X \in A}{\operatorname{argmin}} \rho_{\mu}(X-F) .
$$

Choose functions $h^{1}, \ldots, h^{N}:[0,1] \rightarrow[0,1]$ such that $\sum_{n} h^{n} \equiv 1$ and $h^{n}=0$ outside the set $\left\{\Psi_{\mu^{n}}=\Psi\right\}$. Consider

$$
\begin{aligned}
f^{n}(x) & =\int_{0}^{x} h^{n}(f(y)) d y, \quad x \in \mathbb{R}, \\
Y_{*}^{n} & =f^{n}\left(X_{*}-F+\bar{V}(F)\right),
\end{aligned}
$$

where $f$ is the distribution function of $X_{*}-F+\bar{V}(F)$ and $\int_{0}^{x}$ is the oriented integral. Then $X_{*}, Y_{*}^{1}, \ldots, Y_{*}^{N}$ is a superreplication strategy.

(ii) If the support of each $\mu^{n}$ is $[0,1]$, then $X_{*}$ (if it exists) is unique and any superreplication strategy has the form described above.

Remark. A geometric recipe for finding $X_{*}$ can be found in [13, Subsect. 2.5].

In most typical situations, the sets $\left\{\Psi_{\mu^{n}}=\Psi\right\}$ are disjoint intervals (some of them might be empty) and $X_{*}-F$ has a continuous distribution. Let $\left\{\Psi_{\mu^{n}}=\Psi\right\}=\left[a^{n}, b^{n}\right]$. Then

$$
f^{n}(x)=\int_{0}^{x} I\left(f(y) \in\left[a^{n}, b^{n}\right]\right) d y=\int_{0}^{x} I\left(y \in\left[f^{-1}\left(a^{n}\right), f^{-1}\left(b^{n}\right)\right] d y .\right.
$$

In this case the form of $Y_{*}^{n}$ (see Figure 2) is very similar to the structure of a CDO (for the definition of this contract, see [22, Sect. 9.1.3]).

3. Empirical estimation. The above example provides rather an explicit solution of the superreplication problem for the case, where each $\mathcal{V}^{n}$ is the determining set of Weighted V@R. However, as shown by Table 1, there exist many other natural examples of valuation measures. Here we will consider possible ways to estimate $\bar{V}(F)$ empirically. The empirical estimation of $\bar{V}(F)$ directly by (5.1) might be problematic because it may be hard to capture the set $\left(\bigcap_{n} \mathcal{V}^{n}\right) \cap \mathcal{R}$. Therefore, instead of trying to estimate $\bar{V}(F)$, we will provide an upper estimate of this value. By (5.1),

$$
\bar{V}(F) \leq \inf _{n=1, \ldots, N} \sup \left\{\mathrm{E}_{\mathrm{Q}} F: \mathrm{Q} \in \mathcal{V}^{n} \cap \mathcal{R}\right\} .
$$

The intersection $\mathcal{V}^{n} \cap \mathcal{R}$ is still rather an unpleasant object. However, we can get rid of $\mathcal{R}$ using the following equality whose proof can be found in [13, Th. 2.6]:

$$
\sup \left\{\mathrm{E}_{\mathrm{Q}} F: \mathrm{Q} \in \mathcal{V}^{n} \cap \mathcal{R}\right\}=\inf _{X \in A} \sup \left\{\mathrm{E}_{\mathrm{Q}}(F-X): \mathrm{Q} \in \mathcal{V}^{n}\right\}
$$




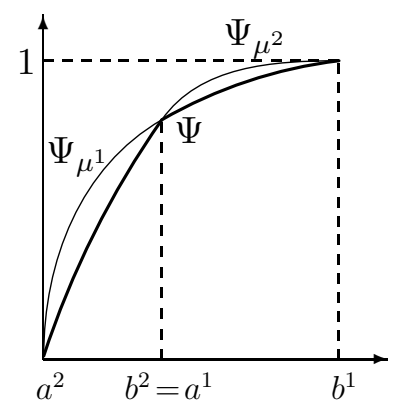

Figure 1

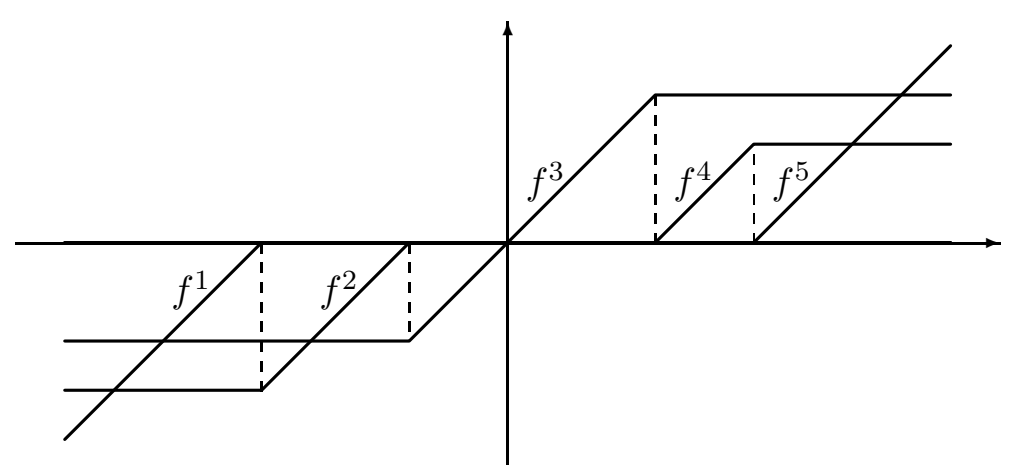

Figure 2. The form of $f^{n}$

As we are trying to find an upper estimate of $\bar{V}(F)$, we need not find this infimum, but can just take the minimum over several $X \mathrm{~s}$.

In the example below, we discuss how to find/estimate $\sup \left\{\mathrm{E}_{\mathrm{Q}} X: \mathrm{Q} \in \mathcal{V}\right\}$ (to simplify the notation, we have replaced $F-X$ by $X$ ) for the valuation measures from Table 1.

Example 5.6. (i) If $\mathcal{V}=\mathcal{D}_{\mu}$, then

$$
\sup \left\{\mathrm{E}_{\mathrm{Q}} X: \mathrm{Q} \in \mathcal{V}\right\}=\rho_{\mu}(-X)
$$

A theoretical representation of $\rho_{\mu}$ is provided by (2.2). Its empirical estimate is provided by (2.4).

(ii) If $\mathcal{V}=\mathrm{E}\left(\mathcal{D}_{\mu} \mid Y\right)$, then, according to (3.1),

$$
\sup \left\{\mathrm{E}_{\mathrm{Q}} X: \mathrm{Q} \in \mathcal{V}\right\}=\rho_{\mu}^{f}(-X ; Y)=\rho_{\mu}(-f(X))
$$

where $f(y)=\mathrm{E}(X \mid Y=y)$. Thus, we should calculate $f$ and then apply the procedures of (i).

(iii) If $\mathcal{V}=\mathcal{X}_{\mathcal{D}_{\mu}}(W)$ and $W$ has a continuous distribution, then, according to Example 3.1 (i),

$$
\sup \left\{\mathrm{E}_{\mathrm{Q}} X: \mathrm{Q} \in \mathcal{V}\right\}=\mathrm{E}_{\mathrm{Q}_{\mu}(W)} X=\mathrm{E} \psi_{\mu}(W) X=\rho_{\mu}^{c}(-X ; W)
$$

In order to find an empirical estimate of this quantity, take time series $\left(x_{1}, w_{1}\right), \ldots,\left(x_{T}, w_{T}\right)$ for $(X, W)$. As the unit time interval here equals the duration of the contingent claim we are trying to price, i.e. it has the order of several months, the ordinary time series might not be available, and one could use the bootstrap technique. Let $w_{(1)}, \ldots, w_{(T)}$ be the numbers $w_{1}, \ldots, w_{T}$ in the increasing order and let $n(i)$ be the 
number such that $w_{(i)}=w_{n(i)}$. Then, according to Example 3.1(ii), an empirical estimate of $\rho_{\mu}^{c}(-X ; W)$ is given by

$$
\sum_{t=1}^{T} x_{n(t)} \int_{(t-1) / T}^{t / T} \psi_{\mu}(x) d x .
$$

(iv) If $\mathcal{V}=\mathcal{X}_{\mathrm{E}\left(\mathcal{D}_{\mu} \mid Y\right)}(W)$, then, according to Example 3.2 and (3.2),

$$
\begin{aligned}
\sup \left\{\mathrm{E}_{\mathrm{Q}} X: \mathrm{Q} \in \mathcal{V}\right\} & =\sup \left\{\mathrm{E}_{\mathrm{Q}} f(Y): \mathrm{Q} \in \mathcal{V}\right\} \\
& =\sup \left\{\int_{\mathbb{R}^{d}} f(y) \varphi(y) \widetilde{\mathrm{P}}(d y): \varphi \in \widetilde{\mathcal{X}}_{\widetilde{\mathcal{D}}_{\mu}}(g)\right\} \\
& =\rho_{\mu}^{c}(f(Y) ; g(Y)) \\
& =\rho_{\mu}^{f c}(X ; Y ; W),
\end{aligned}
$$

where $f(y)=\mathrm{E}(X \mid Y=y)$ and the other notation is introduced in Example 3.2, Thus, we should calculate $f$ and $g$ and then apply the procedures of (iii).

(v) If $\mathcal{V}=\operatorname{conv}_{k} \mathcal{V}^{k}$, then

$$
\sup \left\{\mathrm{E}_{\mathrm{Q}} X: \mathrm{Q} \in \mathcal{V}\right\}=\max _{k} \sup \left\{\mathrm{E}_{\mathrm{Q}} X: \mathrm{Q} \in \mathcal{V}^{k}\right\}
$$

Remark. If $\mathcal{V}=\mathcal{X}_{\mathcal{D}}(W)=\{\mathrm{Q}\}$, where $\mathcal{D}$ is the determining set of a risk measure $\rho$, then $\mathrm{E}_{\mathrm{Q}} F$ is the price of $F$ obtained through the coherent optimality pricing technique of [13; Subsect. 3.3], i.e. it is the number $x$ such that

$$
\inf _{h \in \mathbb{R}} \rho(W+h(F-x))=\rho(W) .
$$

In other words, this is the coherent reservation price of $F$.

The paper [15] contains more information on the empirical estimates of $\rho, \rho^{f}, \rho^{c}$, and $\rho^{f c}$. In particular, it describes convenient Monte Carlo estimation procedures for Alpha V@R and Beta V@R.

4. Liquidity. The definition below was given in [13] following the ideas of 9 .

Definition 5.7. The upper and lower price functions of a contingent claim $F$ are defined as

$$
\begin{aligned}
& \bar{V}(F, v)=\inf \{x \in \mathbb{R}: \exists X \in A \text { such that } X+v(-F+x) \in \mathcal{A}\}, \quad v>0, \\
& \underline{V}(F, v)=\sup \{x \in \mathbb{R}: \exists X \in A \text { such that } X+v(F-x) \in \mathcal{A}\}, \quad v>0 .
\end{aligned}
$$

From the financial point of view, $v$ means the volume of a trade.

If $A$ is a cone, which means that there are no liquidity effects, then $\bar{V}(F, v) \equiv \bar{V}(F)$, $\underline{V}(F, v) \equiv \underline{V}(F)$. However, if $A$ is bounded in a certain sense, then the upper and lower price functions are not constant. In view of the equality $\underline{V}(F, v)=-\bar{V}(-F, v)$, it is sufficient to study only the properties of $\bar{V}(F, v)$.

Theorem 5.8. Let $F \in L_{s}^{1}$.

(i) The function $\bar{V}(F, \cdot)$ is increasing and continuous.

(ii) We have

$$
\lim _{v \downarrow 0} \bar{V}(F, v)=\sup _{\mathrm{Q} \in\left(\bigcap_{n} \mathcal{V}^{n}\right) \cap \mathcal{R}} \mathrm{E}_{\mathrm{Q}} F .
$$


(iii) We have

$$
\lim _{v \rightarrow \infty} \bar{V}(F, v) \leq \sup _{\mathrm{Q} \in \bigcap_{n} \mathcal{V}^{n}} \mathrm{E}_{\mathrm{Q}} F .
$$

If $\sup _{X \in A, Q \in \bigcap_{n} \mathcal{\nu}^{n}}\left|\mathrm{E}_{\mathrm{Q}} X\right|<\infty$, then

$$
\lim _{v \rightarrow \infty} \bar{V}(F, v)=\sup _{Q \in \bigcap_{n} \mathcal{\nu}^{n}} \mathrm{E}_{Q} F .
$$

This statement follows from the results of [13, Subsect. 2.8].

\section{Sensitivity Coefficients}

In this section, we adjust the above technique to measure sensitivity coefficients. The basic idea is as follows. The market is valuing any contingent claim by a valuation measure $Q$. We do not know $Q$ completely, but we can indicate a set $\mathcal{V}$, to which it belongs. For example, the arguments of Section [5 say that it should belong to $\mathcal{V}:=\left(\bigcap_{n} \mathcal{V}^{n}\right) \cap \mathcal{R}$. Suppose moreover that the payoff function of a contingent claim has the form $F=f(S, \xi)$, where $S$ is the value of the underlying asset, with respect to which we wish to take the sensitivity coefficient, and $\xi$ is a random variable that does not depend on $S$ (natural examples are given below). Then we can say that the sensitivity coefficient is

$$
\Delta=\frac{\partial}{\partial S} \mathrm{E}_{\mathrm{Q}} f(S, \xi)=\mathrm{E}_{\mathrm{Q}} \frac{\partial f}{\partial S}(S, \xi) .
$$

Recalling that we do not know $Q$ completely, but we know only $\mathcal{V}$, we can define the interval for deltas as

$$
I(\Delta):=\left\{\mathrm{E}_{\mathrm{Q}} \frac{\partial f}{\partial S}(S, \xi): \mathrm{Q} \in \mathcal{V}\right\} .
$$

The problem of finding/estimating this interval is exactly the problem we have considered above with $F$ replaced by $\frac{\partial f}{\partial S}$.

If the model is complete (like the Black-Scholes-Merton one), then $\mathcal{V}=\left\{Q_{0}\right\}$ and $\mathrm{E}_{\mathrm{Q}_{0}} f(S, \xi)=\varphi(S)$ is the fair price of $F$. In this case

$$
I(\Delta)=\left\{\mathrm{E}_{\mathrm{Q}_{0}} \frac{\partial f}{\partial S}(S, \xi)\right\}=\left\{\varphi^{\prime}(S)\right\},
$$

which is the traditional sensitivity. Thus, in complete models we have one fair price and one sensitivity; in incomplete models we have an interval of fair prices and an interval for sensitivities. In particular, risk in incomplete models cannot be completely eliminated by the delta hedging.

Example 6.1. Let $F$ be the standard call option, i.e. $F=e^{-r T}\left(S_{T}-K\right)^{+}$, where $S_{T}$ is the price of some asset at the expiration date $T$ and $r$ is the risk-free rate. It is natural to model $S_{T}$ as $S \xi$, where $S$ is the current price of the asset and $\xi$ is a random variable whose distribution does not depend on $S$. Then $f(S, \xi)=e^{-r T}(S \xi-K)^{+}$and

$$
\frac{\partial f}{\partial S}(S, \xi)=e^{-r T} \xi I(\xi \geq K / S) .
$$


Example 6.2. Let $F$ be the option on a bond providing the amounts $c_{1}, \ldots, c_{N}$ at future dates $T_{1}<\cdots<T_{N}$. The expiration date of the option is $T<T_{1}$. Suppose that the yield curve evolves as $r(t, T)=r_{t}+\varphi(T-t)$, where $r_{t}$ is a random short rate and $\varphi$ is a fixed shape (for example, this is the case for the Vasicek model). Then the discounted payoff of the option is

$$
F=e^{-T r(0, T)}\left(\sum_{n=1}^{N} c_{n} f_{n}\left(r_{t}\right)-K\right)^{+}
$$

where

$$
f_{n}(r)=\exp \left\{-\left(T_{n}-T\right) r-\left(T_{n}-T\right) \varphi\left(T_{n}-T\right)\right\} .
$$

If we again model $r_{t}$ as $r \xi$, where $r$ is the current short rate and the distribution of $\xi$ does not depend on $r$, then $F=f(r, \xi)$ and

$$
\frac{\partial f}{\partial r}(r, \xi)=e^{-T r(0, T)} \sum_{n=1}^{N} c_{n} f_{n}^{\prime}(r \xi) I\left(\sum_{n=1}^{N} c_{n} f_{n}(r \xi) \geq K\right) .
$$

\section{Summary and Conclusion}

In this paper, we propose a technique for pricing and hedging in incomplete markets that yields finer price intervals than the technique proposed in [9]. The basic idea is: to hedge a liability means to employ a trading strategy and then split the resulting liability into several contracts that can be sold to different groups at the price 0 . The corresponding interval of fair prices is

$$
I(F)=\left\{\mathrm{E}_{\mathrm{Q}} F: \mathrm{Q} \in\left(\bigcap_{n} \mathcal{V}^{n}\right) \cap \mathcal{R}\right\} .
$$

Here $\mathcal{V}^{n}$ is the set of valuation measures used by the $n$-th representative agent (for example, these agents are large companies) or a group of agents. A pleasant feature of this technique, which is not shared by the technique of [9], is: the more groups are taken into account, the smaller are the fair price intervals.

The intersection $\left(\bigcap_{n} \mathcal{V}^{n}\right) \cap \mathcal{R}$ is nonempty provided that there exists no trade involving all the groups, after which each group is better off (Fundamental Theorem of Asset Pricing).

For the estimation purposes, we can replace (7.1) by a wider interval

$$
\bigcap_{n=1}^{N}\left\{\mathrm{E}_{\mathrm{Q}} F: \mathrm{Q} \in \mathcal{V}^{n} \cap \mathcal{R}\right\}
$$

For typical choices of valuation measures (see Table 1), the latter interval admits simple empirical estimation procedures (see Example [5.6).

The interval (17.1) can be made very small if we know the portfolios of the representative agents. If the $n$-th agent is using the expected utility to assess the quality of his/her position, then his/her valuation measure is $c U^{\prime}\left(W_{1}\right) \mathrm{P}$, where $U$ is the utility function and $W_{1}$ is the terminal wealth. If the agent is employing the coherent risk/utility measurement, then a substitute for the above measure is the extreme measure. If, however, we have no information on the structure of the agents' portfolios, then a natural choice of the set of valuation measures is the determining set of some coherent risk measure. Both for the risk measurement purposes and for the estimation of fair price intervals, it is convenient to use not pure coherent risk measures, but rather their factor versions introduced in [15]. 
Furthermore, we define the interval of sensitivities of a contingent claim $F=f(S, \xi)$ with respect to the price $S$ of an underlying asset as

$$
I(\Delta)=\left\{\mathrm{E}_{\mathrm{Q}} \frac{\partial f}{\partial S} f(S, \xi): \mathrm{Q} \in\left(\bigcap_{n} \mathcal{V}^{n}\right) \cap \mathcal{R}\right\} .
$$

If we manage to make $\left(\bigcap_{n} \mathcal{V}^{n}\right) \cap \mathcal{R}$ small, then both the fair price intervals and the sensitivity intervals are small; in particular, the risk of holding $F$ can be successfully eliminated by the delta hedging. 


\section{References}

[1] C. Acerbi. Spectral measures of risk: a coherent representation of subjective risk aversion. Journal of Banking and Finance, 26 (2002), No. 7, p. 1505-1518.

[2] C. Acerbi. Coherent representations of subjective risk aversion. In: G. Szegö (Ed.). Risk measures for the 21st century. Wiley, 2004, p. 147-207.

[3] C. Acerbi, D. Tasche. On the coherence of expected shortfall. Journal of Banking and Finance, 26 (2002), No. 7, p. 1487-1503.

[4] P. Artzner, F. Delbaen, J.-M. Eber, D. Heath. Thinking coherently. Risk, 10 (1997), No. 11, p. 68-71.

[5] P. Artzner, F. Delbaen, J.-M. Eber, D. Heath. Coherent measures of risk. Mathematical Finance, 9 (1999), No. 3, p. 203-228.

[6] P. Barrieu, N. El Karoui. Inf-convolution of risk measures and optimal risk transfer. Finance and Stochastics, 9 (2005), No. 2, p. 269-298.

[7] P. Barrieu, N. El Karoui. Pricing, hedging and optimally designing derivatives via minimization of risk measures. Preprint, available at: http://www . cmap.polytechnique.fr/preprint.

[8] A. Bernardo, O. Ledoit. Gain, loss, and asset pricing. Journal of Political Economy, 108 (2000), No. 1, p. 144-172.

[9] P. Carr, H. Geman, D. Madan. Pricing and hedging in incomplete markets. Journal of Financial Economics, 62 (2001), No. 1, p. 131-167.

[10] P. Carr, H. Geman, D. Madan. Pricing in incomplete markets: from absence of good deals to acceptable risk. In: G. Szegö (Ed.). Risk measures for the 21st century. Wiley, 2004, p. 451-474.

[11] A. Černý, S. Hodges. The theory of good-deal pricing in incomplete markets. In: Mathematical Finance - Bachelier Congress 2000. H. Geman, D. Madan, S. Pliska, T. Vorst (Eds.). Springer, 2001, p. 175-202.

[12] A.S. Cherny. Pricing with coherent risk. Preprint, available at: http://mech.math.msu.su/ cherny.

[13] A.S. Cherny. Equilibrium with coherent risk. Preprint, available at: http://mech.math.msu.su/ ${ }^{\sim}$ cherny.

[14] A.S. Cherny. Weighted V@R and its properties. To be published in Finance and Stochastics. Available at: http://mech.math.msu.su/ ${ }^{\sim}$ cherny.

[15] A.S. Cherny, D.B. Madan. Coherent measurement of factor risks. Preprint, available at: http://mech.math.msu.su/ cherny.

[16] J.H. Cochrane, J. Saá-Requejo. Beyond arbitrage: good-deal asset price bounds in incomplete markets. Journal of Political Economy, 108 (2000), No. 1, p. 79-119. 
[17] F. Delbaen. Coherent risk measures on general probability spaces. In: K. Sandmann, P. Schönbucher (Eds.). Advances in finance and stochastics. Essays in honor of Dieter Sondermann. Springer, 2002, p. 1-37.

[18] F. Delbaen. Coherent monetary utility functions. Preprint, available at http://www.math.ethz.ch/ delbaen under the name "Pisa lecture notes".

[19] H. Föllmer, A. Schied. Stochastic finance. An introduction in discrete time. 2nd Ed., Walter de Gruyter, 2004.

[20] S. Jaschke, U. Küchler. Coherent risk measures and good deal bounds. Finance and Stochastics, 5 (2001), No. 2, p. 181-200.

[21] E. Jouini, W. Schachermayer, N. Touzi. Optimal risk sharing for law invariant monetary utility functions. Preprint, available at:

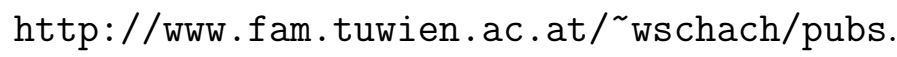

[22] A. McNeil, R. Frey, P. Embrechts. Quantitative risk management: concepts, techniques, and tools. Princeton, 2005.

[23] A. Schied. Risk measures and robust optimization problems. Lecture notes of a minicourse held at the 8th symposium on probability and stochastic processes. Preprint, available on request at: http://www.math.tu-berlin.de/ ${ }^{\text {schied. }}$ 